\title{
Epithelial-Mesenchymal Transition Drives Three-Dimensional Morphogenesis in Mammalian Early Development
}

\author{
Galym Ismagulov ${ }^{\dagger}$, Sofiane Hamidi ${ }^{\dagger}$ and Guojun Sheng * \\ International Research Center for Medical Sciences (IRCMS), Kumamoto University, Kumamoto, Japan
}

OPEN ACCESS

Edited by:

Silvia Garagna,

University of Pavia, Italy

Reviewed by:

Maria Cecilia Cirio,

CONICET Institute of

Physiology, Argentina

Isabelle Migeotte,

Université libre de Bruxelles, Belgium

Alexandre Francou,

Sloan Kettering Institute, United States

*Correspondence:

Guojun Sheng

sheng@kumamoto-u.ac.jp

†These authors have contributed equally to this work

Specialty section:

This article was submitted to

Cell Growth and Division,

a section of the journal

Frontiers in Cell and Developmental

Biology

Received: 08 December 2020

Accepted: 06 January 2021

Published: 11 February 2021

Citation:

Ismagulov G, Hamidi S and Sheng G

(2021) Epithelial-Mesenchymal Transition Drives Three-Dimensional

Morphogenesis in Mammalian Early

Development.

Front. Cell Dev. Biol. 9:639244.

doi: 10.3389/fcell.2021.639244
From fertilization to onset of gastrulation, a mammalian embryo goes through several rounds of cellular morphogenesis resembling phenomena of epithelial-mesenchymal transition (EMT) and mesenchymal-epithelial transition (MET), collectively referred to as EMTs. How these EMT events play a role in shaping the three-dimensional (3-D) architecture of the developing embryo is not well-understood. In this review, we present a model in which cellular morphogenesis, represented primarily by dynamic changes in its epithelialization status, is the driving force of embryonic 3-D organization. This is achieved through the integration of three key components of mammalian early development, the pluripotency regulation, morphogenetic signaling, and biomechanical force anisotropy. Although cells in an early embryo do not exhibit full mesenchymal characteristics, our model underscores the importance of investigating molecular regulation of epithelial cell polarity and partial EMT/MET in understanding mammalian early development.

Keywords: EMT, epiblast, pluripotency, polarity, gastrulation

\section{INTRODUCTION}

After fertilization, a mammalian embryo undergoes several rounds of early cleavages before acquisition of apicobasal polarity through compaction and segregation of inner and outer cells through asymmetric divisions (Humiecka et al., 2017). Cells inheriting the apical cortical domain at 8-cell to 16-cell division will reestablish polarity and position themselves in the outer layer of the embryo and will be biased to become trophectoderm (TE) progenitors. The rest of the blastomeres lack apical surface and apicobasal polarity and will be positioned in the inner part of the embryo and eventually contribute to the inner cell mass (ICM) (Maitre et al., 2016). The ICM cells will then sort out, in an actin-dependent manner, to give rise to an epithelialized primitive endoderm (hypoblast) (Bedzhov and Zernicka-Goetz, 2014), whereas the remaining epiblast precursors maintain their non-epithelial characteristics. Those epiblast precursors will then undergo an MET process to form an apicobasally polarized epiblast epithelium and generate a pro-amniotic cavity as a consequence of this process. The epithelialized epiblast subsequently goes through an EMT process during gastrulation, generates the mesoderm and definitive endoderm, and lays down the foundation of the three-germ layer and three-dimensional (3-D) mammalian body plan.

Up till the onset of gastrulation, the architecture of a mammalian embryo largely reflects morphogenetic features of its constituent cells, whereas more complex interactions involving cells, tissues, and tissue compartments govern gastrulation and post-gastrulation morphogenesis. In this review, we aim to synthesize published data on early mammalian development with respect to 
the regulation of cellular morphogenesis and an embryo's 3-D organization, with an emphasis on the interplay between epithelial (E) and/or mesenchymal (M) status, pluripotency, biomechanical forces and morphogen signaling.

\section{INTER-DEPENDENCY BETWEEN CELLULAR MORPHOLOGY AND PLURIPOTENCY}

Cellular potency is defined by its ability to differentiate into various cell types either in vivo or in vitro, varying from totipotency, corresponding to the status of blastomeres before lineage segregation between the TE and ICM, to unipotency which corresponds to cells that can differentiate only into a single cell type (Figure 1). Status of a cell's potency in early development reflects both the embryo's developmental stage and the cell's morphogenetic status. For instance, loss of totipotency of early blastomeres is associated with segregation of TE and ICM cells, and the restriction of potency is more pronounced for the outer, apicobasally polarized cells (TE) than the non-polarized ICM cells, which remain pluripotent and can give rise to all cell lineages in an adult body.

Pluripotency was conceptualized based on differentiation capacity (either in vitro or in vivo) of cells isolated in vivo and maintained in vitro. Recent efforts have been made to associate pluripotency with features of either the original developmental stage during pluripotent cell derivation or the putative developmental stage after those pluripotent cells have achieved a steady state under a given culture condition. Four pluripotency states have so far been characterized using cell morphological and molecular criteria. The first one is termed "naïve pluripotency," which corresponds to the ICM cell after TE specification and segregation. Naive pluripotent cells do not have apicobasal polarity. They express a particular set of associated genes (e.g., REX1) (Ghimire et al., 2018). The second type is termed "formative pluripotency," which corresponds to epithelializing epiblast precursors at the time of primitive endoderm specification and segregation. The formative pluripotent cells retain germline differentiation capability and are defined molecularly by OTX2 upregulation (Stumpf and MacArthur, 2019; Kinoshita et al., 2020). The third type is "primed pluripotency," corresponding to a partially epithelialized epiblast prior to gastrulation. These cells have many epithelial characteristics (although they are not fully epithelialized) and have lost the capacity to contribute to the germline or to make chimeras (a phenomenon likely related to their epithelial status). Primed pluripotent cells have a differentiation bias toward specific cell linages that reflects their developmental progression along the antero-posterior axis of the embryo (Shahbazi et al., 2017). Finally, the fourth stage is the "pluripotency exit." Cells at this stage are on the verge of differentiation (Thakurela et al., 2019) and are characterized by a progressive reduction of the core pluripotency factors such as OCT4, NANOG and SOX2. These cells can still reverse their pluripotent status upon changes in culture conditions (Hamidi et al., 2020) or developmental cues (Tian et al., 2019).
In human development, these transitions are also supported by progressive epithelialization of the epiblast and its segregation into the embryonic epiblast (giving rise to future three-germ layers) and the amniotic epiblast (contributing to the ectoderm portion of the amnion), and the appearance of the proamniotic cavity. The molecular mechanisms that link changes in cellular morphology and cellular potency are still not well-explored. GRHL2, an epithelial state inducer, has been reported to control the expression of a subset of the pluripotent network genes upon transition from naive to primed pluripotency state (Chen et al., 2018). Conversely, MCRS1, a mesenchymal state inducer (Liu et al., 2014), is required to maintain the epiblast lineage after trophectoderm and primitive endoderm specification, suggesting that the ability to retain an non-polar mesenchymallike phenotype in the ICM is critical (Cui et al., 2020).

\section{CELL MORPHOLOGY AND BIOMECHANICAL FORCES}

Biomechanical forces in the embryo are a function of two main cellular features: cell motility and cell deformation. They play critical roles in shaping the embryo's 3-D architecture prior to gastrulation. During the process of TE and ICM segregation (Marikawa and Alarcón, 2009), mechanical forces are directly responsible for their position and fate specification through regulation of the orientation of asymmetric divisions. Cells located on the embryo's surface retain contractile abilities and differentiate into TE cells, whereas cells on the center, without contractile abilities, become the ICM (Bissiere et al., 2018). Consecutively, during blastocyst cavity formation, trophectoderm epithelial integrity associated with directional fluid transport results in the establishment of a blastocyst luminal pressure that drives further lumenogenesis and embryo growth (Wang et al., 2018; Chan et al., 2019).

Non-polarized naïve epiblast precursors at this stage do not respond to mechanical stimuli (Verstreken et al., 2019). Later on, at the peri-implantation stage, upon ECM-mediated epiblast epithelialization, the luminal pressure will induce apical surface repulsion, which contributes to the fusion of multiple rosettelike clusters and to the establishment of inverted cup shapedmorphology of the mouse embryo (Christodoulou et al., 2018; Dokmegang et al., 2020). In parallel, the progression of epiblast cells from non-polarized naïve pluripotent cells to apicobasally polarized primed cells enables biomechanical signaling to play a role in epiblast cells' fate decisions (Verstreken et al., 2019). An amniote embryo's shape at the peri-gastrulation stage exhibit species-specific variation (Sheng, 2015). As a consequence, the underlying mechanical forces and their interplay with cellular morphogenesis can also vary. In the chick embryo, for example, where the peri-gastrulation embryo's shape can be likened to a disc, a supracellular actomyosin ring located in the marginal zone, at the edge of the embryonic tissue, controls the embryo global geometry. This ring also induces cellular deformations that are transmitted locally though the epiblast epithelial structure, inducing "polonaise-like" coordinated cellular migration and initiation of the primitive streak (Saadaoui et al., 2020). The 


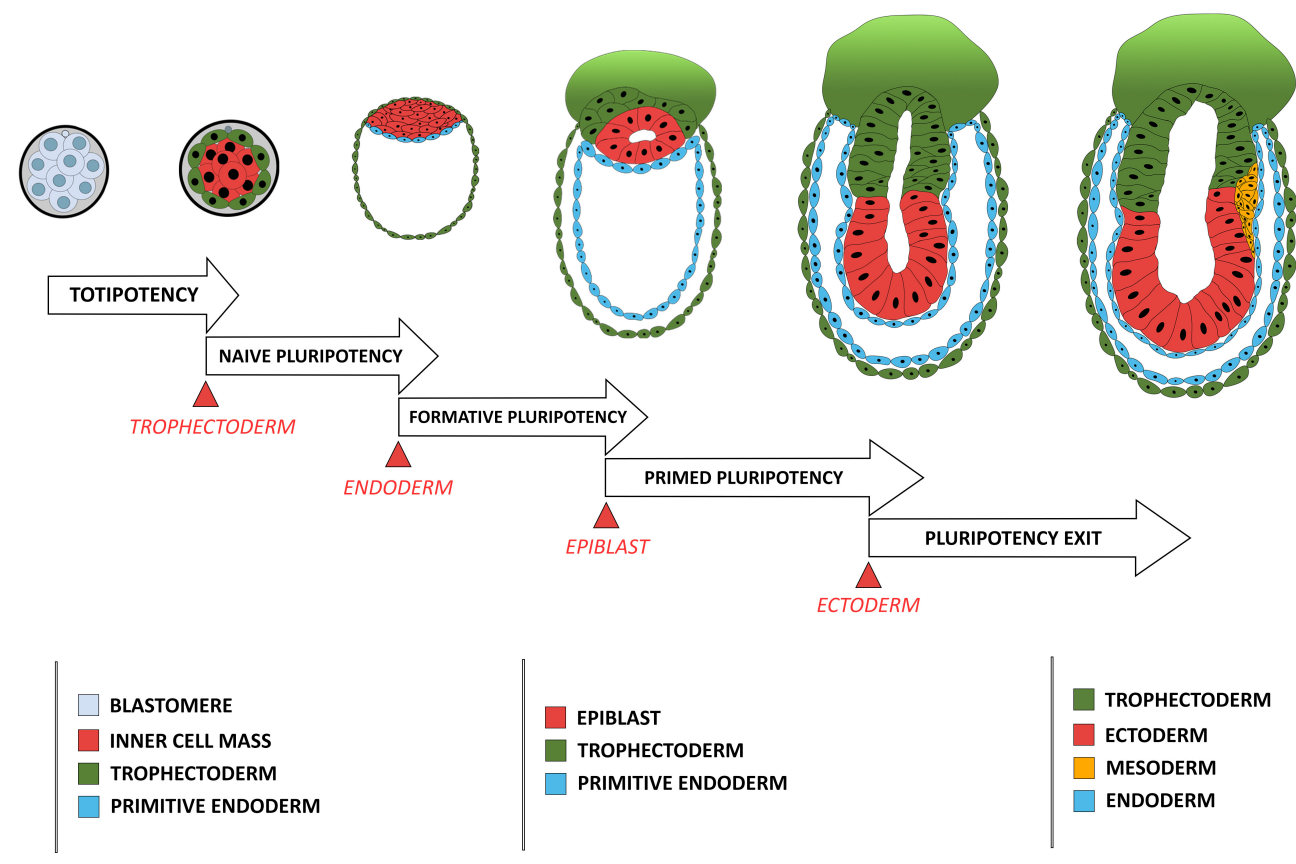

FIGURE 1 | Comparison between the developmental stage of a mouse embryo and the concomitant restriction in cellular potency. Each restriction in cellular potency is associated with the creation of an embryonic structure through an epithelialization process: the totipotency is lost when the trophectoderm epithelializes; the naïve pluripotency loss corresponds to the epithelialization of the endoderm; the formative state loss is concomitant with the epithelialization of the epiblast; and the primed pluripotency loss corresponds to the acquisition of a fully epithelial organization by the epiblast leading to pluripotency exit, a stage poised for differentiation including ectoderm-fated epithelium formation and mesendoderm induction through another round of EMT.

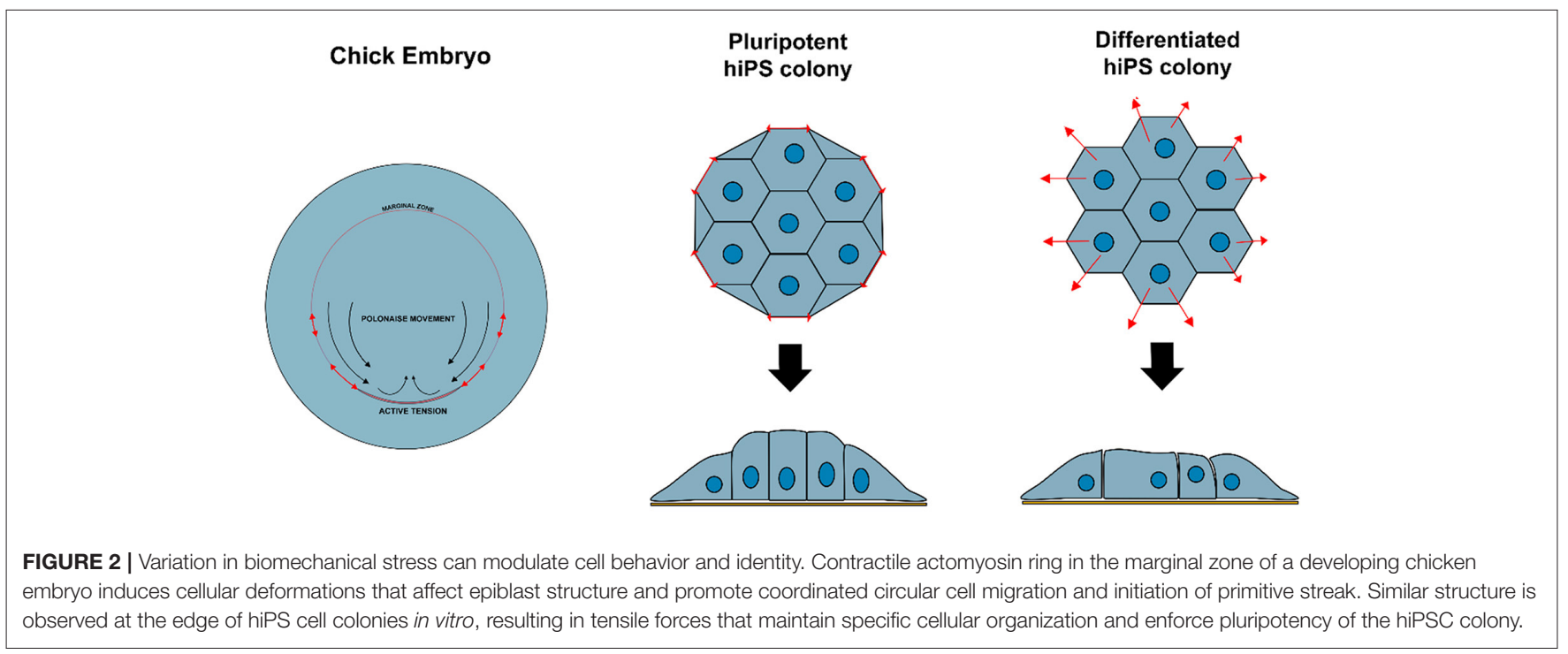

"global" architecture of the pre-gastrulating chick embryo is somewhat similar to the "embryonic disc" seen in some mammalian groups, including in the human, and interestingly, a similar supracellular actin structure has also been reported to exist at the edge of disc-shaped human pluripotent stem cell (PSC) colonies in vitro (Närvä et al., 2017) (Figure 2). However, its existence in vivo (in the human embryo) or its causal relationship with human primitive streak initiation is unclear.
In vitro findings suggest that human PSCs can adopt a spectrum of morphological identities between fully epithelial and partially mesenchymal in vitro, as a consequence of differences in ECM compositions and concentrations (Hamidi et al., 2020), possibly reflecting ECM-dependent localized variations in migratory potential and epithelialization status of epiblast cells in vivo (Nakaya et al., 2013). It has been reported that basement membrane composition can influence PSC's epithelialization 

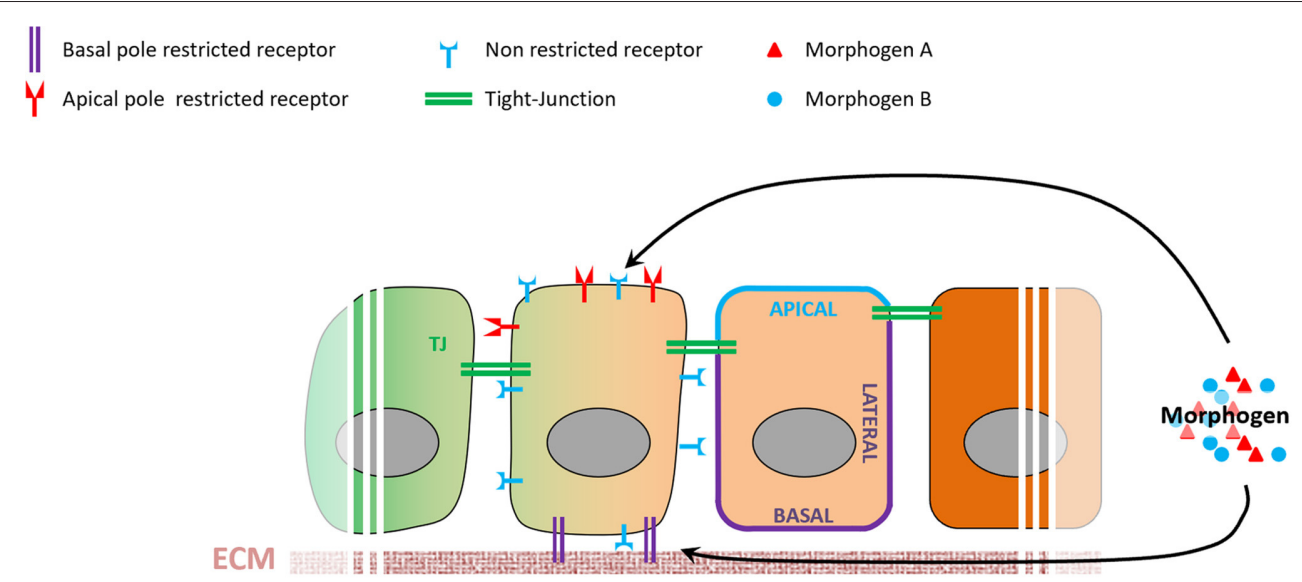

MESENCHYMAL
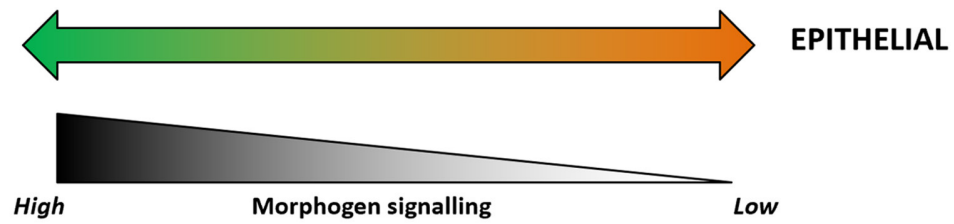

FIGURE 3 | Variations in epithelial organization can modulate levels of cellular response to a given morphogen. During the epithelialization process, tight junctions act as impermeable seals that compartmentalize the apical and basolateral membranes of a cell. As a consequence, a morphogen present at the apical pole will not be able to reach receptors localized on the basolateral membrane, and vice-versa. Some receptors are known to be localized specifically to either the apical or basolateral plasma membranes, and variations in cell's epithelial organization will result in different levels of signaling pathway activation.

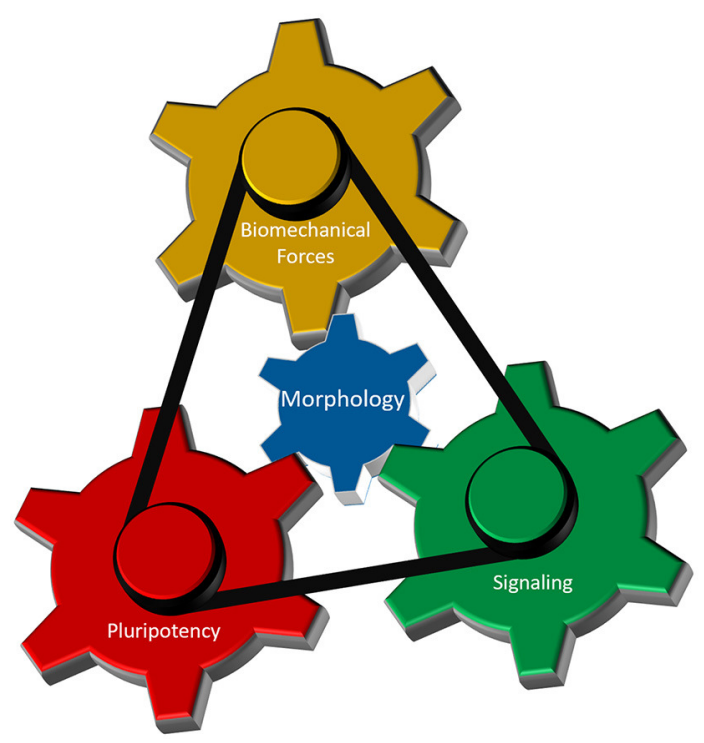

FIGURE 4 | Cellular morphology as the fundamental parameter that coordinates different regulatory components of 3-D morphogenesis. Biomechanical forces, cellular potency and molecular signaling are the main components that dictate early morphogenesis of an embryo. Cellular morphology, defined by its epithelial and/or mesenchymal status, interacts with each of those three components to induce local variations in cellular responses necessary for the formation of highly reproducible structure in an early embryo.

(Futaki et al., 2019; Hamidi et al., 2020) or induce EMT upon basement membrane breakdown (Nakaya et al., 2008), consistent with the fact that ECM's heterogeneity increases as embryonic development proceeds (Loganathan et al., 2016; Futaki et al., 2019). Spatiotemporal-specific ECM composition can be the blueprint of biomechanical force anisotropy and of an embryo's 3-D structure by providing a highly-specific local environment adequate for generating biomechanics-mediated signaling complexity (Loganathan et al., 2014).

\section{CELL MORPHOLOGY AND RESPONSE TO MORPHOGENS}

Morphogen signaling regulates many aspects of embryonic development (Tuazon and Mullins, 2015), action of which remains poorly understood. For instance, cells exposed to the same signals exhibit distinct fates depending on their spatiotemporal information in the embryo (Morgani and Hadjantonakis, 2020), and cellular geometry controls the level of response to morphogens in the mouse embryo (Zhang et al., 2019). Epithelialization-associated specification of apical and basolateral compartments of a cell involves the establishment of apical junctions as barriers to molecular diffusion. Morphogen availability and morphogen receptors are known to localize preferentially to either the apical or the basolateral membranes of the epithelium (Yin et al., 2017), resulting in a likewise compartmentalized molecular and subcellular response to a given morphogen (Figure 3). BMP receptors, for example, are present in mesodermal cells at levels similar to those in epiblast cells, but the BMP signaling activity is higher in the mesoderm cells (Morgani et al., 2018). Transition between epithelial and mesenchymal states of the same cell population (e.g., EMT during gastrulation) involves loss of apicobasal polarization, which may 


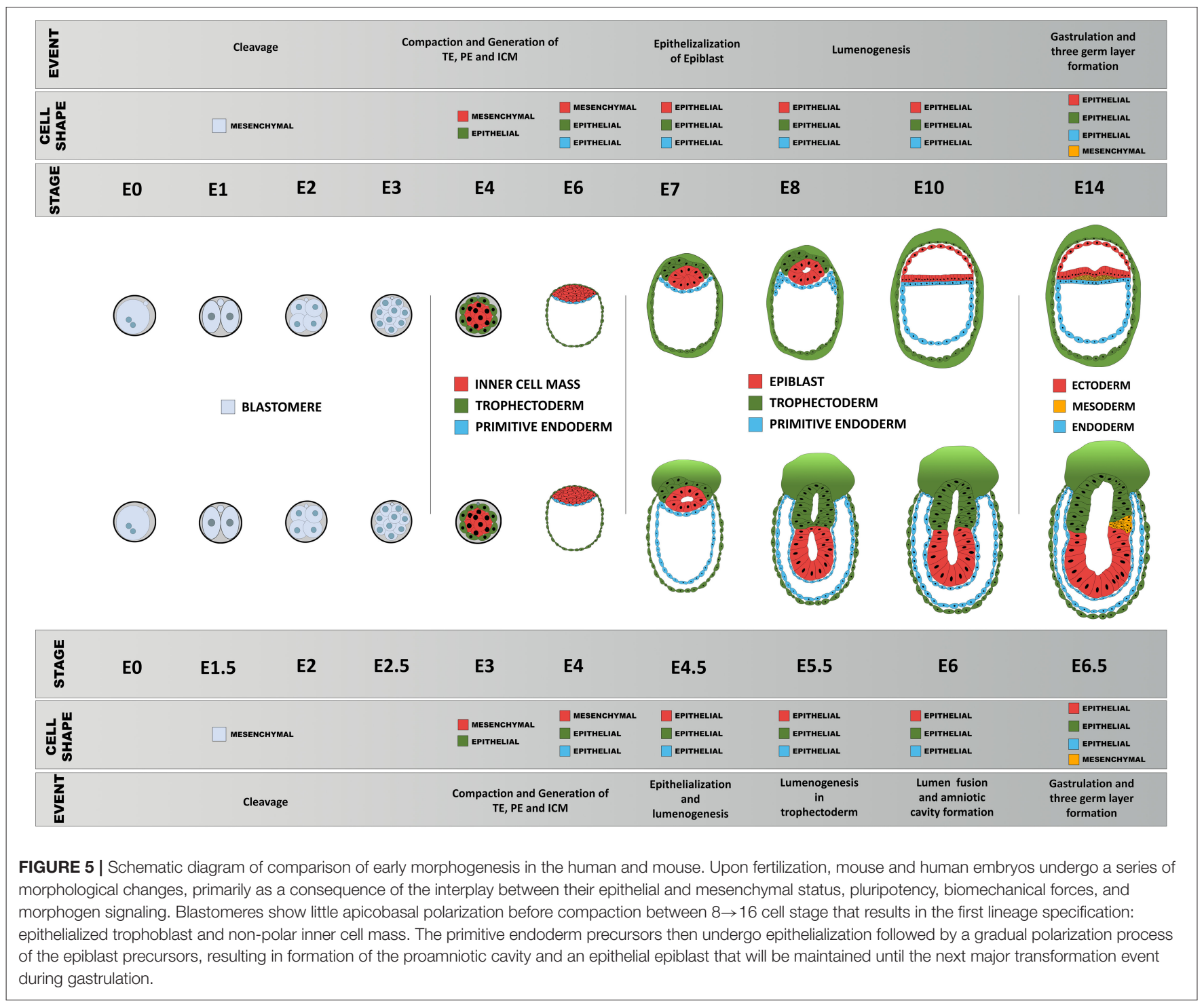

lead to more BMP receptors being exposed to BMP morphogen in mesoderm cells and enhance cellular response to it. In vitro models of human epiblast formation and differentiation allow us to investigate this question in more detail (Warmflash et al., 2014; Williams and Solnica-Krezel, 2017; Hamidi et al., 2020). In hESC colonies, differences in receptor accessibility due to apicobasal polarization led to the establishment of a gradient of BMP signaling activation, resulting in differential commitment of PSCs to the three germ layers (Etoc et al., 2016; Hamidi et al., 2020).

Additional levels of complexity in modulating differential effect of morphogen on cellular fate specification can be achieved by ECM heterogeneity and its role in mediating morphogen availability or receptor activation at the basolateral side. In addition to controlling cells' epithelial or mesenchymal status, the ECM can also act as a filtering mesh that can slow down, concentrate or biochemically modulate the action of a given morphogen. As a consequence, cells with similar morphological characteristics and morphogen exposure can display different responses depending on their ECM composition.

\section{WHAT CAN WE LEARN FROM THIS EMT/MET-CENTRIC MODEL?}

As discussed above, interplay between cellular morphology, pluripotency, biomechanical forces, and morphogen signaling is critical for establishing proper 3-D architecture of a developing embryo (Figure 4). However, the mechanistic relationship between these events remains elusive. Why do cells require concurrent morphological transitions in order to achieve pluripotency changes? Do mechanical forces affect morphogen signaling and pluripotency levels, or is it the signaling that results in a specific manifestation of mechanical forces that in turn regulate cellular behavior? According to a recent 
model (Das et al., 2019), biomechanical forces can be a secondary consequence of short-range morphogen signaling that ensures the robustness and long-range transmission of information necessary for proper embryo-level morphogenesis. To accomplish this, cells are required to have appropriate cellcell and cell-ECM interactions, permitting transmission of the mechanical signals. Thus, cellular morphology may act as a signal integrator that can receive, process, and send specific information among cells located in neighboring tissues.

Indeed, large-scale morphogenetic movements (e.g., during primitive streak formation) involve oriented cellular intercalation and morphology changes that can be understood from the perspective of polarized cytoskeletal organization and directional biomechanical tension (Ferro et al., 2020), associated with the supracellular organization of the actin-myosin cytoskeletal network of the epiblast epithelium (Hamidi et al., 2019; Ferro et al., 2020). However, in view of the robustness and speed of signal transduction through mechanical means, it is still unclear how cells can sense the requirement for morphological changes and effectively communicate with each other to construct elaborate, supracellular structures. A possible hypothesis, from analysis of zebrafish gastrulation, could be that there is heterogeneity in such integration and that some cells respond and relay more readily a given signal to their neighbors (Das et al., 2019).

During early embryonic development, it is unclear why cells need to undergo successive morphological transformation in order to achieve the complexity in cellular differentiation. Is it a necessity to ensure proper control over the morphogenetic process or just a direct consequence of morphology changes? The former would imply that it is necessary to restrict cellular potency as embryonic morphogenesis progresses in order to reduce potential differentiation errors. However, this paradigm seems to be contradicted by a recent study on totipotency loss and trophectoderm differentiation in which the authors showed that ICM-derived naive PSCs retain the ability to differentiate into the trophectoderm lineage (Guo et al., 2020). The latter implies that acquisition of epithelial features is sufficient to trigger differentiation process(es) that would restrict their potency. This scenario may be a more realistic one as a recent study showed that attachment of the cellular plasma

\section{REFERENCES}

Bedzhov, I., and Zernicka-Goetz, M. (2014). Self-organizing properties of mouse pluripotent cells initiate morphogenesis upon implantation. Cell 156, 1032-1044. doi: 10.1016/j.cell.2014.01.023

Bergert, M., Lembo, S., Sharma, S., Russo, L., Milovanović, D., Gretarsson, K. H., et al. (2020). Cell surface mechanics gate embryonic stem cell differentiation. Cell Stem Cell. 28, 1-8. doi: 10.1016/j.stem.2020.10.017

Bissiere, S., Gasnier, M., Alvarez, Y. D., and Plachta, N. (2018). Cell fate decisions during preimplantation mammalian development. Curr. Top. Dev. Biol. 128, 37-58. doi: 10.1016/bs.ctdb.2017.11.001

Chan, C. J., Costanzo, M., Ruiz-Herrero, T., Mönke, G., Petrie, R. J., Bergert, M., et al. (2019). Hydraulic control of mammalian embryo size and cell fate. Nature 571, 112-116. doi: 10.1038/s41586-019-1309-x membrane to the submembrane cortex protects naïve PSCs from progressing to a primed state or further (Bergert et al., 2020). Using Ezrin mutant proteins to modulate the membrane-tocortex attachment (MCA), the authors showed that a high MCA inhibited PSC differentiation, whereas a low MCA resulted in a permissive state that allows differentiation. Moreover, strong membrane-to-cortex attachment is known to maintain cellular polarity and protect cells from morphologic transition (Houk et al., 2012; Schneider et al., 2013). This suggests that in the case of MCA, cells are preferentially using physical forces as checkpoints to control their progression through important morphogenetic events (Figure 5). This and other similar hypotheses could be tested in the future by combining in vitro models of early epiblast-like PSC organization (Deglincerti et al., 2016) with the use of biomaterials capable of dynamic modulation of their physical properties (LeValley and Kloxin, 2019). Such approaches would enable a powerful in vitro platform, with high reproducibility and programmability, to investigate how mechanical signals are integrated into the biochemical network that regulates cellular specification and tissue patterning and whether cell morphological changes might be the missing link reconciling how mechanical and chemical signals influence cell identity during development.

\section{DATA AVAILABILITY STATEMENT}

The original contributions presented in the study are included in the article/supplementary material, further inquiries can be directed to the corresponding author/s.

\section{AUTHOR CONTRIBUTIONS}

GS conceived the manuscript topic. GI, SH, and GS discussed about the writing and wrote the manuscript. All authors agreed on the content of manuscript.

\section{FUNDING}

This work has been partly funded by a MEXT scholarship to GI and Kakenhi Kiban B and C, JST, and Takeda foundation research grafts to GS.

Chen, A. F., Liu, A. J., Krishnakumar, R., Freimer, J. W., DeVeale, B., and Blelloch, R. (2018). GRHL2-dependent enhancer switching maintains a pluripotent stem cell transcriptional subnetwork after exit from naive pluripotency. Cell Stem Cell 23, 226-238.e224. doi: 10.1016/j.stem.2018.06.005

Christodoulou, N., Kyprianou, C., Weberling, A., Wang, R., Cui, G., Peng, G., et al. (2018). Author correction: sequential formation and resolution of multiple rosettes drive embryo remodelling after implantation. Nat. Cell Biol. 20:1434. doi: 10.1038/s41556-018-0245-6

Cui, W., Cheong, A., Wang, Y., Tsuchida, Y., Liu, Y., Tremblay, K. D., et al. (2020). MCRS1 is essential for epiblast development during early mouse embryogenesis. Reproduction 159, 1-13. doi: 10.1530/REP-19-0334

Das, D., Jülich, D., Schwendinger-Schreck, J., Guillon, E., Lawton, A. K., Dray, N., et al. (2019). Organization of embryonic morphogenesis via mechanical information. Dev. Cell 49, 829-839.e825. doi: 10.1016/j.devcel.2019.05.014 
Deglincerti, A., Croft, G. F., Pietila, L. N., Zernicka-Goetz, M., Siggia, E. D., and Brivanlou, A. H. (2016). Self-organization of the in vitro attached human embryo. Nature 533, 251-254. doi: 10.1038/nature17948

Dokmegang, J., Yap, M. H., Han, L., Cavaliere, M., and Doursat, R. (2020). Computational modelling unveils how epiblast remodelling and positioning rely on trophectoderm morphogenesis during mouse implantation. doi: 10.1101/2020.06.08.140269

Etoc, F., Metzger, J., Ruzo, A., Kirst, C., Yoney, A., Ozair, M. Z., et al. (2016). A balance between secreted inhibitors and edge sensing controls gastruloid self-organization. Dev. Cell 39, 302-315. doi: 10.1016/j.devcel.2016.09.016

Ferro, V., Chuai, M., McGloin, D., and Weijer, C. J. (2020). Measurement of junctional tension in epithelial cells at the onset of primitive streak formation in the chick embryo via non-destructive optical manipulation. Development 147:dev175109. doi: 10.1242/dev.175109

Futaki, S., Nakano, I., Kawasaki, M., Sanzen, N., and Sekiguchi, K. (2019). Molecular profiling of the basement membrane of pluripotent epiblast cells in post-implantation stage mouse embryos. Regene. Therap. 12, 55-65. doi: 10.1016/j.reth.2019.04.010

Ghimire, S., Van der Jeught, M., Neupane, J., Roost, M. S., Anckaert, J., Popovic, M., et al. (2018). Comparative analysis of naive, primed and ground state pluripotency in mouse embryonic stem cells originating from the same genetic background. Sci. Rep. 8:5884. doi: 10.1038/s41598-018-24051-5

Guo, G., Stirparo, G. G., Strawbridge, S., Spindlow, D., Yang, J., Clarke, J., et al. (2020). Human naïve epiblast cells possess unrestricted lineage potential. doi: $10.1101 / 2020.02 .04 .933812$

Hamidi, S., Nakaya, Y., Nagai, H., Alev, C., Kasukawa, T., Chhabra, S., et al. (2020). Mesenchymal-epithelial transition regulates initiation of pluripotency exit before gastrulation. Development 147:dev184960. doi: 10.1242/dev.184960

Hamidi, S., Nakaya, Y., Nagai, H., Alev, C., Shibata, T., and Sheng, G. (2019). Biomechanical regulation of EMT and epithelial morphogenesis in amniote epiblast. Phys. Biol. 16:041002. doi: 10.1088/1478-3975/ab1048

Houk, A. R., Jilkine, A., Mejean, C. O., Boltyanskiy, R., Dufresne, E. R., Angenent, S. B., et al. (2012). Membrane tension maintains cell polarity by confining signals to the leading edge during neutrophil migration. Cell 148, 175-188. doi: 10.1016/j.cell.2011.10.050

Humiecka, M., Szpila, M., Kło,ś, P., Maleszewski, M., and Szczepańska, K. (2017). Mouse blastomeres acquire ability to divide asymmetrically before compaction. PLoS ONE 12:e0175032. doi: 10.1371/journal.pone.0175032

Kinoshita, M., Barber, M., Mansfield, W., Cui, Y., Spindlow, D., Stirparo, G. G., et al. (2020). Capture of mouse and human stem cells with features of formative pluripotency. Cell Stem Cell. doi: 10.1016/j.stem.2020.11.005

LeValley, P. J., and Kloxin, A. M. (2019). Chemical approaches to dynamically modulate the properties of synthetic matrices. ACS Macro Lett. 8, 7-16. doi: 10.1021/acsmacrolett.8b00808

Liu, M. X., Zhou, K. C., and Cao, Y. (2014). MCRS1 overexpression, which is specifically inhibited by miR-129*, promotes the epithelial-mesenchymal transition and metastasis in non-small cell lung cancer. Mol. Cancer 13:245. doi: 10.1186/1476-4598-13-245

Loganathan, R., Little, C. D., Joshi, P., Filla, M. B., Cheuvront, T. J., Lansford, R., et al. (2014). Identification of emergent motion compartments in the amniote embryo. Organogenesis 10, 350-364. doi: 10.4161/org.36315

Loganathan, R., Rongish, B. J., Smith, C. M., Filla, M. B., Czirok, A., Bénazéraf, B., et al. (2016). Extracellular matrix motion and early morphogenesis. Development 143, 2056-2065. doi: 10.1242/dev.127886

Maître, J. L., Turlier, H., Illukkumbura, R., Eismann, B., Niwayama, R., Nédélec, F., et al. (2016). Asymmetric division of contractile domains couples cell positioning and fate specification. Nature 536, 344-348. doi: $10.1038 /$ nature 18958

Marikawa, Y., and Alarcón, V. B. (2009). Establishment of trophectoderm and inner cell mass lineages in the mouse embryo. Mol. Reprod. Dev. 76, 1019-1032. doi: $10.1002 / \mathrm{mrd} .21057$

Morgani, S. M., and Hadjantonakis, A. K. (2020). Signaling regulation during gastrulation: insights from mouse embryos and in vitro systems. Curr. Top. Dev. Biol. 137, 391-431. doi: 10.1016/bs.ctdb.2019.11.011

Morgani, S. M., Metzger, J. J., Nichols, J., Siggia, E. D., and Hadjantonakis, A. K. (2018). Micropattern differentiation of mouse pluripotent stem cells recapitulates embryo regionalized cell fate patterning. Elife 7:e32839. doi: 10.7554/eLife.32839.029
Nakaya, Y., Sukowati, E. W., and Sheng, G. (2013). Epiblast integrity requires CLASP and dystroglycan-mediated microtubule anchoring to the basal cortex. J. Cell Biol. 202, 637-651. doi: 10.1083/jcb.201302075

Nakaya, Y., Sukowati, E. W., Wu, Y., and Sheng, G. (2008). RhoA and microtubule dynamics control cell-basement membrane interaction in EMT during gastrulation. Nat. Cell Biol. 10, 765-775. doi: 10.1038/ncb1739

Närvä, E., Stubb, A., Guzmán, C., Blomqvist, M., Balboa, D., Lerche, M., et al. (2017). A strong contractile actin fence and large adhesions direct human pluripotent colony morphology and adhesion. Stem Cell Rep. 9, 67-76. doi: 10.1016/j.stemcr.2017.05.021

Saadaoui, M., Rocancourt, D., Roussel, J., Corson, F., and Gros, J. (2020). A tensile ring drives tissue flows to shape the gastrulating amniote embryo. Science 367, 453-458. doi: 10.1126/science.aaw1965

Schneider, D., Baronsky, T., Pietuch, A., Rother, J., Oelkers, M., Fichtner, D., et al. (2013). Tension monitoring during epithelial-to-mesenchymal transition links the switch of phenotype to expression of moesin and cadherins in NMuMG cells. PLoS ONE 8:e80068. doi: 10.1371/journal.pone.0080068

Shahbazi, M. N., Scialdone, A., Skorupska, N., Weberling, A., Recher, G., Zhu, M., et al. (2017). Pluripotent state transitions coordinate morphogenesis in mouse and human embryos. Nature 552, 239-243. doi: 10.1038/nature24675

Sheng, G. (2015). Epiblast morphogenesis before gastrulation. Dev. Biol. 401, 17-24. doi: 10.1016/j.ydbio.2014.10.003

Stumpf, P. S., and MacArthur, B. D. (2019). Machine learning of stem cell identities from single-cell expression data via regulatory network archetypes. Front. Genet. 10:2. doi: 10.3389/fgene.2019.00002

Thakurela, S., Sindhu, C., Yurkovsky, E., Riemenschneider, C., Smith, Z. D., Nachman, I., et al. (2019). Differential regulation of OCT4 targets facilitates reacquisition of pluripotency. Nat. Commun. 10:4444. doi: 10.1038/s41467-019-11741-5

Tian, T. V., Di Stefano, B., Stik, G., Vila-Casadesús, M., Sardina, J. L., Vidal, E., et al. (2019). Whsc1 links pluripotency exit with mesendoderm specification. Nat. Cell Biol. 21, 824-834. doi: 10.1038/s41556-019-0342-1

Tuazon, F. B., and Mullins, M. C. (2015). Temporally coordinated signals progressively pattern the anteroposterior and dorsoventral body axes. Semin. Cell Dev. Biol. 42, 118-133. doi: 10.1016/j.semcdb.2015.06.003

Verstreken, C. M., Labouesse, C., Agley, C. C., and Chalut, K. J. (2019). Embryonic stem cells become mechanoresponsive upon exit from ground state of pluripotency. Open Biol. 9:180203. doi: 10.1098/rsob.180203

Wang, X., Zhang, Z., Tao, H., Liu, J., Hopyan, S., and Sun, Y. (2018). Characterizing inner pressure and stiffness of trophoblast and inner cell mass of blastocysts. Biophys. J. 115, 2443-2450. doi: 10.1016/j.bpj.2018.11.008

Warmflash, A., Sorre, B., Etoc, F., Siggia, E. D., and Brivanlou, A. H. (2014). A method to recapitulate early embryonic spatial patterning in human embryonic stem cells. Nat. Methods 11, 847-854. doi: 10.1038/nmeth.3016

Williams, M. L., and Solnica-Krezel, L. (2017). Regulation of gastrulation movements by emergent cell and tissue interactions. Curr. Opin. Cell Biol. 48, 33-39. doi: 10.1016/j.ceb.2017.04.006

Yin, X., Kang, J. H., Andrianifahanana, M., Wang, Y., Jung, M. Y., Hernandez, D. M., et al. (2017). Basolateral delivery of the type I transforming growth factor beta receptor is mediated by a dominant-acting cytoplasmic motif. Mol. Biol. Cell 28, 2701-2711. doi: 10.1091/mbc.e17-0 5-0334

Zhang, Z., Zwick, S., Loew, E., Grimley, J. S., and Ramanathan, S. (2019). Mouse embryo geometry drives formation of robust signaling gradients through receptor localization. Nat. Commun. 10:4516. doi: 10.1038/s41467-019-1 2533-7

Conflict of Interest: The authors declare that the research was conducted in the absence of any commercial or financial relationships that could be construed as a potential conflict of interest.

Copyright (c) 2021 Ismagulov, Hamidi and Sheng. This is an open-access article distributed under the terms of the Creative Commons Attribution License (CC BY). The use, distribution or reproduction in other forums is permitted, provided the original author(s) and the copyright owner(s) are credited and that the original publication in this journal is cited, in accordance with accepted academic practice. No use, distribution or reproduction is permitted which does not comply with these terms. 